\title{
Programming integrative multi-enzyme systems and ionic strength for recyclable synthesis of glutathione
}

Xiangwei Cui ${ }^{1}$, Zeyuan Wang ${ }^{1}$, Zonglin $\mathrm{Li}^{1}$, Xing Zhang ${ }^{1}$, Zhimin $\mathrm{Li}^{1,2^{*}}$

1. State Key Laboratory of Bioreactor Engineering, East China University of Science and Technology, 130 Meilong Road, Shanghai 200237, China

2. Shanghai Collaborative Innovation Center for Biomanufacturing Technology, 130

Meilong Road, Shanghai 200237, China

* Corresponding author: Zhimin Li

Telephone: $+86-21-64252095$

E-Mail: $\underline{\text { lizm@ecust.edu.cn }}$ 
Table S1 Primers for co-expression cassettes construction

\begin{tabular}{|c|c|}
\hline primer & Sequence (from5'to3') \\
\hline $\mathrm{C}-\mathrm{F}$ & AAGCTTGCGGCCGCACTCGAGCACCACCACCACCACCACTGAGATCCGGCTGCTA \\
\hline C-R & CGAGCCACCGCCACCCGAGCCACCGCCACCATCAATATGTGCATCGCCTTTGG \\
\hline GshF-F & CGGTGGCTCGGGTGGCGGTGGCTCGATGATGACCATCAATCAGTT \\
\hline GshF-R & GGTGCTCGAGTGCGGCCGCAAGCTTTTAGTCCATTTCCGGAAACAGC \\
\hline $\mathrm{T}-\mathrm{F}$ & GGTGGCGGTGGCTCGGGCGGTGGTG GGTCGGGAGCCCACATCGTGATGGTGGA \\
\hline $\mathrm{T}-\mathrm{R}$ & CATATGGCTGCCGCGCGGCACCAGGCCGCTGCTGTGATGATGATGATGATGGCTG \\
\hline PPK-F & CCTGGTGCCGCGCGGCAGCCATATGATGGCCGGTCCGAGCGCAGA \\
\hline PPK-R & CACCACCGCCCGAGCCACCGCCACCACGATTCAGACGCTGATCCGGATGC \\
\hline PT-F & AAGCTGTTTCCGGAAATGGACTAAAAGCTTTAATACGACTCACTATAGGGGAATTG \\
\hline PT-R & GGTGGTGGTGCTCGAGTGCGGCCGCTTACTTCGTCGGCTTGTAGGCGTCCA \\
\hline $\mathrm{CP}-\mathrm{F}$ & GCGGCCGCACTCGAGCACCACCACCACCACCACTGAGATCCGGCTGCTA \\
\hline $\mathrm{CP}-\mathrm{R}$ & AAGCTTTTAGTCCATTTCCGGAAACAGCTTTGCCAGAATTTTAGGGGTA \\
\hline RT-F & GTTTCCGGAAATGGACTAAAAGCTTTTTGTTTAACTTTAAGAAGGAGATAT \\
\hline $\mathrm{RC}-\mathrm{F}$ & GTTTCCGGAAATGGACTAAAAGCTTTTTGTTTAACTTTAAGAAGGAGATAT \\
\hline $\mathrm{RC}-\mathrm{R}$ & CCTATAGTGAGTCGTATTAAAGCTTTTAGTCCATTTCCGGAAACAGCTTTG \\
\hline PC-F & GTTTCCGGAAATGGACTAAAAGCTTTAATACGACTCACTATAGGGGAATTG \\
\hline PC-R & CTTCTTAAAGTTAAACAAAAAGCTTTTAGTCCATTTCCGGAAACAGCTTTG \\
\hline JT-R & CAGCCGGATCTCAGTGGTGGTGGTTAACGATTCAGACGCTGATCC \\
\hline PG-F & CCACCACCACTGAGATCCGGCTGCTAACAAAG \\
\hline
\end{tabular}


Table S2 Strains and plasmids used in this study

\begin{tabular}{|c|c|c|}
\hline Strains and plasmids & Relevant genotype or phenotype & References \\
\hline \multicolumn{3}{|l|}{ plasmids } \\
\hline pET28a & Cloning vector, $\operatorname{Kan}^{\mathrm{R}}$ & Our lab \\
\hline pET28a-ppk & pET28a carries polyphosphate kinase gene & In this study \\
\hline pET28a-M3 & $\begin{array}{l}\text { pET28a carries the mutant gene of bifunctional } \\
\text { glutathione synthetase }\end{array}$ & In this study \\
\hline pET28a-SpyCatcherM3 & pET28a carries gene cassette SpyCatcherM3 & In this study \\
\hline pET28a-JTSpyTag & pET28a carries gene cassette JTSpyTag & In this study \\
\hline pET28a- SpyCatcherM3- & pET28a carries gene cassette SpyCatcherM3- & In this study \\
\hline JTSpyTag & JTSpyTag & \\
\hline pET28a-M3-JT & pET28a carries gene cassette M3-JT & In this study \\
\hline $\begin{array}{l}\text { pET28a- SpyCatcherM3- } \\
\text { RBSJTSpyTag }\end{array}$ & $\begin{array}{l}\text { pET28a carries gene cassette SpyCatcherM3- } \\
\text { RBSJTSpyTag }\end{array}$ & In this study \\
\hline $\begin{array}{l}\text { pET28a- SpyCatcherM3- } \\
\text { RBS SpyCatcherM3- } \\
\text { JTSpyTag }\end{array}$ & $\begin{array}{l}\text { pET28a carries gene cassette SpyCatcherM3- } \\
\text { RBS SpyCatcherM3- JTSpyTag }\end{array}$ & In this study \\
\hline $\begin{array}{l}\text { pET28a- SpyCatcherM3- } \\
\text { RBS SpyCatcherM3- } \\
\text { RBSJTSpyTag }\end{array}$ & $\begin{array}{l}\text { pET28a carries gene cassette SpyCatcherM3- } \\
\text { RBS SpyCatcherM3- RBSJTSpyTag }\end{array}$ & In this study \\
\hline $\begin{array}{l}\text { pET28a- SpyCatcherM3- } \\
\text { SpyCatcherM3- } \\
\text { RBSJTSpyTag }\end{array}$ & $\begin{array}{l}\text { pET28a carries gene cassette SpyCatcherM3- } \\
\text { SpyCatcherM3- RBSJTSpyTag }\end{array}$ & In this study \\
\hline \multicolumn{3}{|l|}{ Srtains } \\
\hline E.coli BL21(DE3) & $\begin{array}{l}\text { Wild type of Escherichia coli for protein } \\
\text { expression }\end{array}$ & Our lab \\
\hline $\mathrm{CT}$ & $\begin{array}{l}\text { E.coli BL21(DE3) containing plasmid pET28a- } \\
\text { pET28a-SpyCM3-JTSpyT }\end{array}$ & In this study \\
\hline CRT & $\begin{array}{l}\text { E.coli BL21(DE3) containing plasmid pET28a- } \\
\text { pET28a-SpyCM3-RBSJTSpyT }\end{array}$ & In this study \\
\hline CRCT & $\begin{array}{l}\text { E.coli BL21(DE3) containing plasmid pET28a- } \\
\text { SpyCM3-RBSSpyCM3-JTSpyT }\end{array}$ & In this study \\
\hline CRCRT & $\begin{array}{l}\text { E.coli BL21(DE3) containing plasmid pET28a- } \\
\text { SpyCM3-RBSSpyCM3-RBSJTSpyT }\end{array}$ & In this study \\
\hline
\end{tabular}


CCRT

M3-JT
E.coli BL21(DE3) containing plasmid pET28a- In this study SpyCM3-SpyCM3-RBSJTSpyT

E.coli BL21(DE3) containing plasmid pET28a- In this study M3-JT 
Table S3 Estimated charges of peptide SpyCatcher/SpyTag, fusion protein SpyCM3/JTSpyT at $\mathrm{pH} 8.0$

\begin{tabular}{ccccc}
\hline $\mathrm{pH}$ & SpyCatcher & SpyTag & SpyCM3 & JTSpyT \\
\hline 8.0 & -10.6 & 0.5 & -53.2 & 7.8 \\
\hline
\end{tabular}


Fig.S1 The purified enzymes of M3 (lane 1), SpyCM3 (lane2), PPKJT (lane3), JTSpyT (lane4)

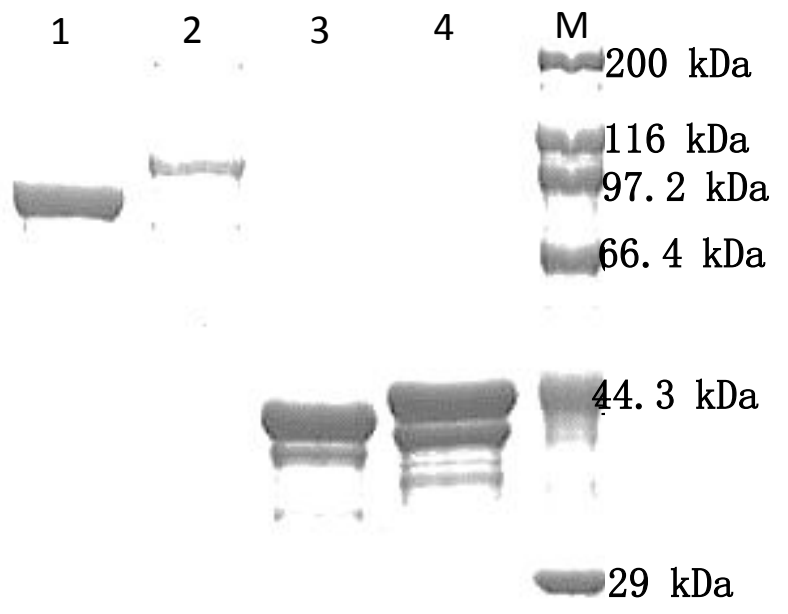


Fig.S2 Comparison of activities and thermostabilities of fusion proteins with M3 and PPKJT as control. A: comparison of enzyme activities; B: comparison of thermostability with PPKJT and JTSpyT; C: comparison of thermostability with M3 and SpyCM3. Each group was repeated three times.
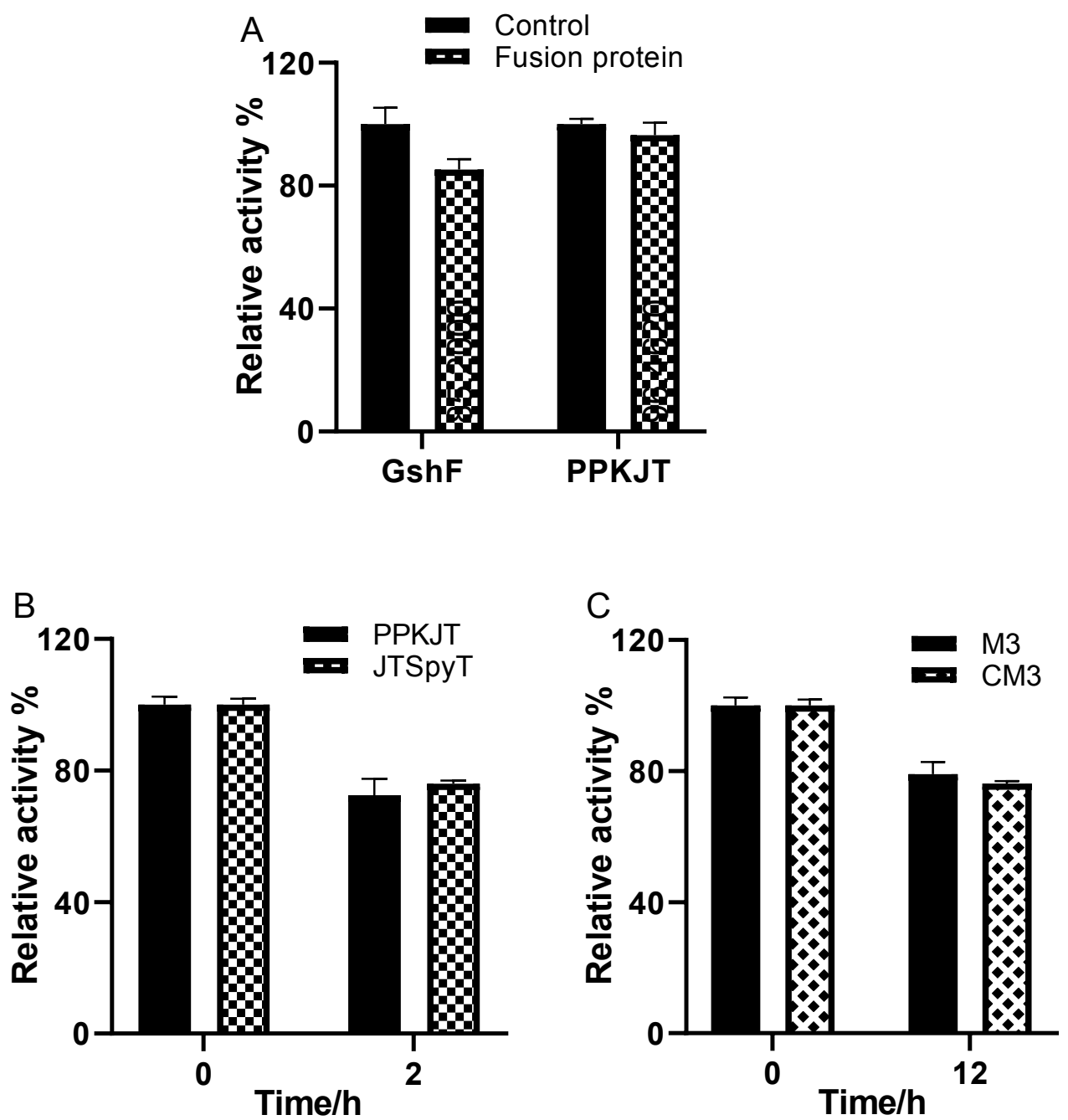
Fig.S3 SDS-PAGE showed the changes of MESs concentration in different incubation time at $45^{\circ} \mathrm{C}$. M: marker, 1: supernatant of $0 \mathrm{~h}, 2$ : supernatant of $1 \mathrm{~h}, 3$ : supernatant of $2 \mathrm{~h}, 4$ : precipitation of $1 \mathrm{~h}, 5$ : precipitation of $2 \mathrm{~h}$

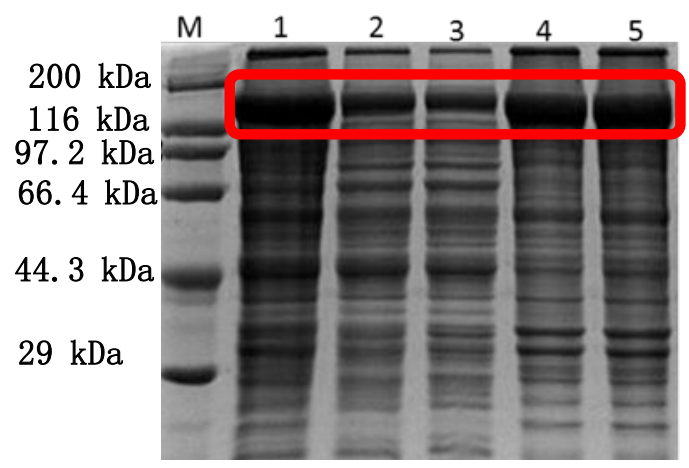


FigS4 Analysis of MESs expression levels with different recombinant strains by SDS-

PAGE, 1-5:CT CRT CCRT CRCT CRCRT.

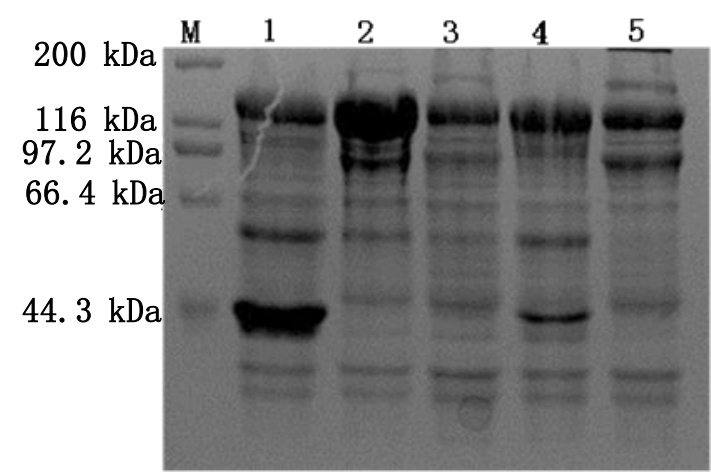


Fig S5 The verification of reuse with CRT and M3-JT (20 mM amino acids). Each group was repeated three times.
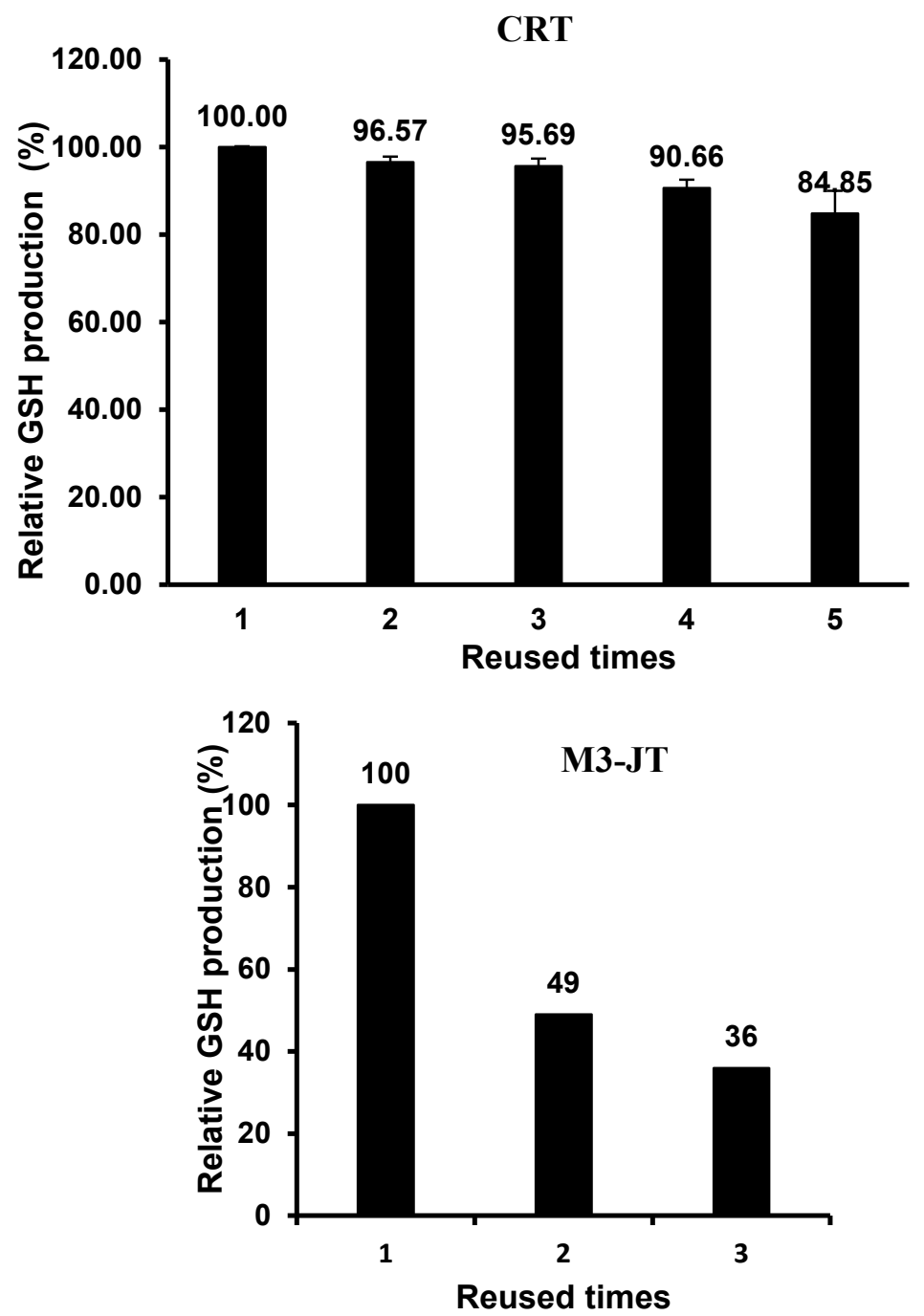
FigureS6 SDS-PAGE analysis of proteins in supernatant during whole-cell recycling.

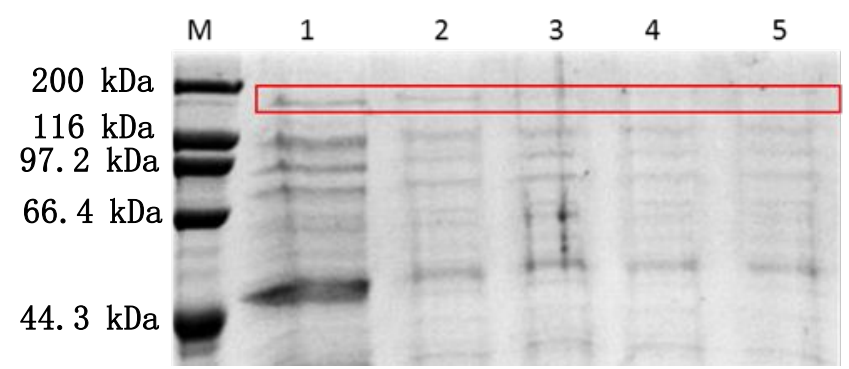

